\title{
Early Detection and Elimination of Underground Pests using IOT
}

\author{
B.Arthi, G.Padmapriya, M.Aruna
}

\begin{abstract}
Agriculture is the backbone of our nation. Our country's growth is highly dependent on the growth of agriculture. Hence, it is mandatory that we need to identify and incorporate new technologies and solutions to protect and improvise problems faced in cultivation by the agriculturalists. In agricultural sector one of the major reasons of losses are due to pests and plant diseases. The harm and impairment caused by insect pests is one of the fundamental factors affecting the crop production. Pests and insects can have adverse effect on agricultural production in turn affecting market access, natural environment, and our lifestyle. The objective of this work is focused on detecting and eliminating the underground worms that causes damage to the crops. An unmanned embedded system is proposed that automatically detects the existence of underground worms in the soil that are not visible to human eyes. This system helps in identifying the existence of worms in the soil using a thermocouple device and eradicates them by instantiating the pesticide on them.
\end{abstract}

Index Terms - IOT, Thermocouple, Sensors, Pest Control, Crop Infection.

\section{INTRODUCTION}

The crop productivity depends on environmental factors and product resources such as temperature, humidity, water, labor and electricity costs. In all these factors, crop disease causes $20-30 \%$ reduction of the productivity due to the crop being infected by pest. Hence, the disease of the crop is considered as one of the critical factors that affects the productivity of the crops [1]. Considering the impact of pests on crop growth, it is important for the farmer to focus on the cause of disease in the crops during its growth. Even though the farmers have started using pesticides and fertilizers at various stages of the crop growth, it has been a challenging task for the farmers in identifying the type of pests and the related disease. Until now, they either rely on the opinion of the experts or their own past experiences when the disease is doubtful. If appropriate actions are not initiated at the right time, it leads to decrease in the yield of the crop. For increased productivity, identification and monitoring of plant disease is required right from the early stage to harvest stage of the crop. During the various stages of the crop growth, effective usage of fertilizers and pesticides are required. Even though there is rise in usage of remote sensing solutions, the visibility and monitoring of underground soil during critical growth stages of crops continues to be a major concern. Fig 1 shows the samples of undersoil worms that affect the crop growth.

Revised Manuscript Received on July 10, 2019.

Dr.B.Arthi, Associate Professor, Department of CSE, Saveetha University, SIMATS, Chennai, T.N, India.

Dr.G.Padmapriya, Associate Professor, Department of CSE, Saveetha University, SIMATS, Chennai, T.N, India.

Dr.M.Aruna, Assistant Professor Department of CSE, Saveetha University, SIMATS, Chennai, T.N, India.

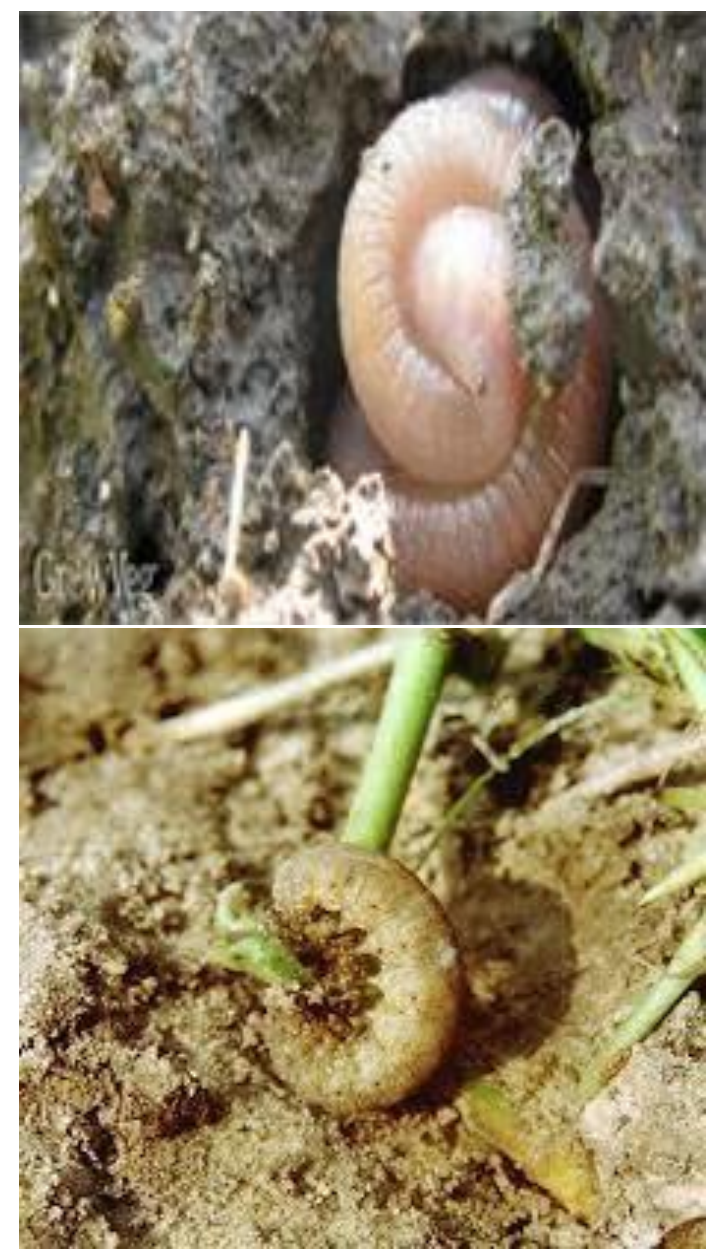

Fig. 1. Worm under Soil that affect the crop growth

One of the real time examples to be quoted is the last year cultivation phase of onion plantation, there was a new issue faced by farmers where the roots of the onion plant was rotten. This was caused by the one category of the underground worm called as white grubs, which in turn affects the onion productivity.

This problem could not be detected by expert farmers or by image processing technique because only after the roots are fully rotten, color of the leaf changes above the soil and also because the white grub resides in the under soil. In this the most critical one need is the identification of underground worms in the soil that affect the plant growth. These underground worms are not visible to human eyes and the presence of these worms affect the growth of crops drastically which ultimately affects the productivity of crop.

Published By

Blue Eyes Intelligence Engineering \& Sciences Publication

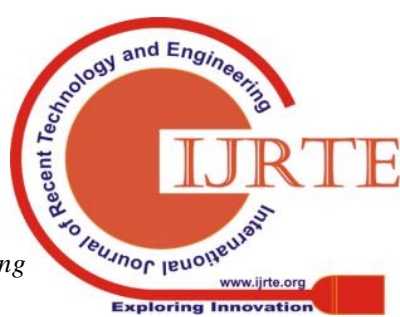


Identification of such worms in the earlier stages would be extremely helpful in avoiding the crop degradation. The proposed model consists of a ground based agricultural robotic vehicle enabled with thermocouple device and a pesticide spray system.

The proposed vehicle is movable, compact with unswerving system that automatically surveys agricultural croplands, and detects worms or insects that damage the crops. Once the presence of worm or insect is identified, the right amount of pesticide on the damaged crop is instigated. For identifying the presence of worm or insect in the soil, a thermocouple enabled in the robotic vehicle is placed at certain depth of soil, nearer to the plant where the thermal level of soil is monitored [10]. Primarily the soil maintains certain range of thermal level. The grubs in the soil has different thermal level. And hence the thermal level of the soil that has grubs or worms will have higher temperature than that of the normal level. Based on the difference in temperature levels adequate pesticides can be sprayed at the affected area. If there is no difference in temperature, then it is concluded that there are no worms and hence fertilizers are sprayed.

\section{RELATED WORKS}

For the progress in agriculture and improved crop productivity, many techniques and models had been introduced. However, disease identification in early stages and their forecasting accuracy is still controversial [5][6]. However, above all, diseases that affect the crops is one of the crucial factors that causes $30-40 \%$ reduction in the overall crop productivity. Thus, the infection caused by the crop disease is the important factor affecting the productivity of the crops [12]. There are many image processing mechanisms available which dynamically analyses the images of the crop and gives the information about the diseases. The farmers can take appropriate actions based on the analysis results [7]. These methodologies perform the diagnosis and prediction of the disease with data set of images using deep learning. Now- a-days there are also expert systems that are available for agricultural operations in assisting the farmers in identifying the appropriate crops for their land. It also helps to carry out efficient and effective crop management and proposes a lot of accurate results for various agricultural factors. Many authors have also carried out a survey on Crop Disease Diagnoses using expert systems. Advancement in agriculture is the remote sensing solutions. eAGROBOT is a robot that was developed to be used for agriculture. This robot was developed to bring solutions to many agricultural problems with satellite communications [1][2]. It is easy to use, easily movable and fault tolerant machine that automatically observes the agricultural land and detect infections or diseases if exists. It is also used to spray a overall understanding and analysis of the agricultural land with the help of data analytics which also helps in decision support [3].

To increase the productivity, it is mandatory to monitor the growth of the crops throughout their life period. Few authors have used image processing techniques to analyze and identify diseases that occur in sunflower plant. This is pesticide for the infected crops. In future, farmers can obtain

done by analyzing the sunflower leaf. The images of the sunflower leaf are taken through a high-resolution camera and they are preprocessed. The preprocessed images are applied to clustering technique to get the retrieve the diseased portion of the leaf.

Various machine learning algorithms are run on the output and are categorized as textures and colors based on the features. To achieve the maximum accuracy a comparison is made on the various machine learning algorithms viz a Multi nominal logistic regression, KNearest Neighbors, Naïve Bayes and Multi support Vector Machine. Recently for digital images, a segmentation technique using region growing algorithms are used. The distinguishing of disease spot and grey level are done by using determinate or fixed criterion of the region growing algorithm [8]. But in reality, the images of the diseased leaf have unclear edges of the object and are fuzziness. The researchers have proposed an adaptive segmentation algorithm by integrating local threshold and seeded region growing (LTSRG) to improve the disease spot in the leaf images [9]. The researchers have used pixels from segmentation algorithm that ascertains the higher gray level in R-channel than G-Channel as initial points (Pixels) and each connected seed region are ascertained using local threshold $\mathrm{C}$ [11]. The threshold $\mathrm{C}$ is recalculated by including new seed pixel. The calculation is done until no seed pixel can be included. All the above-mentioned work is used to detect or identify the crop diseases above the ground level [4]. Systems with underground visibility for early detection of worms that affect the root of the crop are a challenge.

\section{METHODOLOGY}

The following are the hardware for the embedded system and the processer that make up the system.

\section{A. Embedded Hardware Requirements}

The primary components of the system are IC16F877A microcontroller, temperature sensor known as thermocouple, driver circuit and LCD display. The soil temperature is measured using a sensor. In case if the temperature sensor range crosses the threshold value it will be alerted by buzzer. This helps in the detection of worm under the soil as early as possible.

\section{B. Hardware Description}

PIC16F877A: The performance of PIC16F877A machine is highly reliable and efficient. It has a RISC CPU. It works based on 35 simple word instructions. Its operating speed is $200 \mathrm{MHz}$ measured as clock input and it has an instruction cycle of 200nS. PIC16F877A has a RAM data memory capacity of $368 \times 8$ bit and an EEPROM data memory capacity of $256 \times 8$. Apart from all these, it has a flash memory capacity of $8 \mathrm{k} \times 14$. The voltage range for operating PIC16F877A is $2.0-5.56$ volts. It also has a 2.8 bit timer and along with a 16 bit timer. PIC16F877A has a 10 bit multi-channel Analog to Digital converter Synchronous Serial Port (SSP) with SPI 
(master code) and I2C (master/slave). Its EEPROM memory has a 1000000 times erase/write cycle for data.

Power Supply Circuit: A power supplied of 5 volts is needed for the hardware included for this framework. The major microcontroller gives the power to all the devices that are interfaced.

LCD: Liquid Crystal Display is an electronic display used by many applications. Most of the devices and the circuits uses a 16x2 LCD display and it acts as a primary module. This type of screen can display 16 characters for each and line and there are two lines available. Command registers and Data registers are used in this display. In such displays each character is displayed as $5 \times 7$ pixel matrix.

Driver Circuit: A driver may be a circuit or a component in electrical or electronics that helps in taking control of other circuit or component, such as a high-power transistor or liquid crystal display (LCD). The major use of these driver circuits is to manage the power flow through the circuit. It also helps to balance other devices in the circuit.

Transformer: Fig 2 shows the essential model of the transformer. The potential electrical device can step down the facility and provide voltage of 0-230 volts to 0-6 volts level. Then the secondary of the potential electrical device are going to be connected to the exactitude rectifier, which is built with the assistance of op-amp. The benefits of exploitation exactitude rectifier area unit offer peak voltage output as DC; remainder of the circuits will offer solely RMS output.

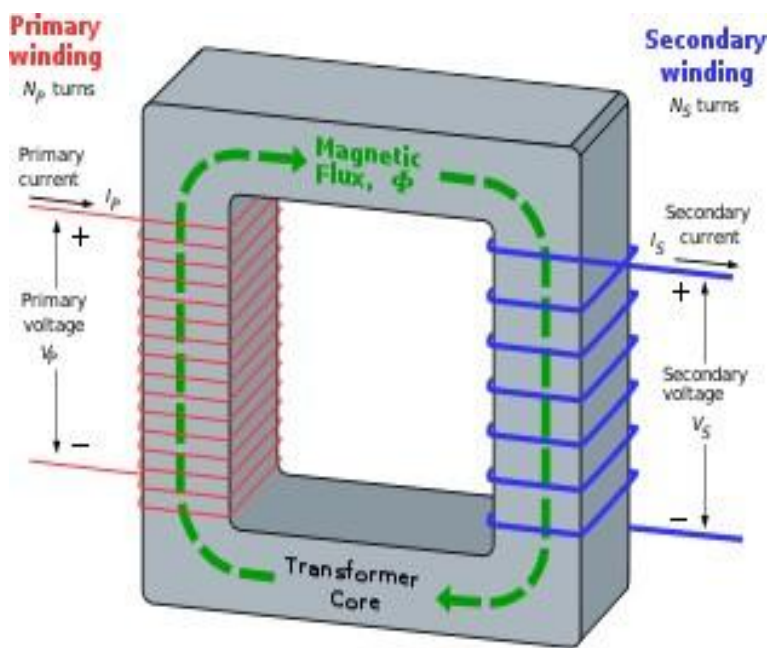

Fig.2. Basic model of transformer

Bridge Rectifier: A Bridge Rectifier is a circuit that is connected with four diodes. The following fig 3 gives the representation of bridge rectifier

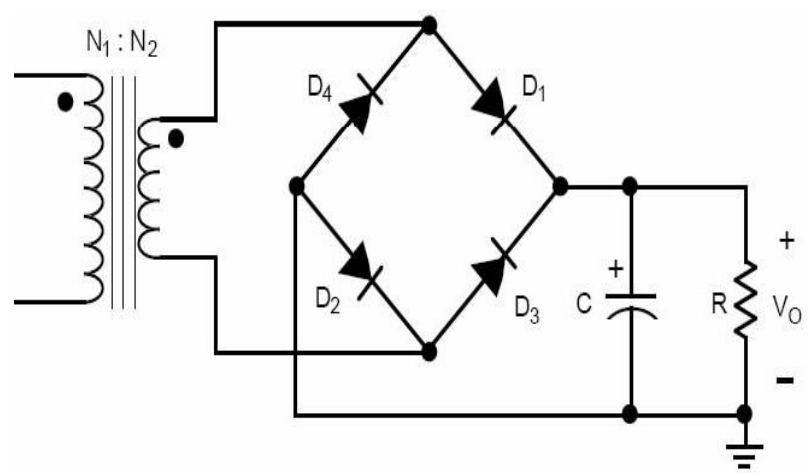

Fig.3. Four diode bridge rectifier

The contribution to the circuit is connected to the slantingly inverse corners of the system, and furthermore the yield is taken from the rest of the 2 corners. This plan enables us to expect that the electrical gadget is working appropriately and there's a positive potential, at reason An and a negative potential at reason $\mathrm{B}$. the positive potential at reason A can advance inclination D3 and invert predisposition D4. The negative potential at reason B can advance predisposition D1 and invert D2. At now D3 and D1 square measure forward one-sided and can allow current stream to experience them; D4 and D2 square measure turn around one-sided and can square current stream.

The way for flow stream is from reason B through D1, up through RL, through D3, through the optional of the electrical gadget back to reason $\mathrm{B}$. this way is shown by the strong bolts. Waveforms (1) and (2) are regularly found crosswise over D1 and D3.

One-half cycle later the extremity over the auxiliary of the electrical gadget invert, forward biasing D2 and D4 and turn around biasing D1 and D3.Current stream can as of now be from reason A through $\mathrm{D} 4$, up through $\mathrm{RL}$, through D2, through the optional of T1, and back to reason A. This way is shown by the messed up bolts. Waveforms (3) and (4) is found crosswise over D2 and D4. This move through RL is regularly inside a similar bearing. In coursing through RL this current builds up a voltage appreciate that indicated wave structure (5). Since current moves through the heap (RL) all through each [*fr1] cycles of the connected voltage, this leeway rectifier might be a rectifier. One bit of leeway of an extension rectifier over a standard rectifier is that with a given electrical gadget the scaffold rectifier creates a voltage yield that is about twofold that of the conventional full-wave circuit.

IC Voltage Regulators: Voltage regulators comprise a category of wide used ICs. Regulator IC units contain the electronic equipment for reference supply, comparator electronic equipment, management device, and overload protection dead one IC. IC units offer regulation of either a set positive voltage, a set negative voltage, or Associate in Nursing adjustably set voltage. The regulators may be chosen for operation with load currents from many milli amperes to tens of amperes, akin to power ratings from milli watts to tens of watts. 
Firstly, A three-terminal transformer has an unfettered DC input voltage, $\mathrm{Vi}$, which is been applied to 1 input terminal. Next, controlled DC output voltage, Vo, from a second terminal, with the third terminal connected to ground. The sequence of seventy eight regulators bid straddling positive structured voltages from 5 to 24 volts. Similarly, the series seventy nine regulators offer straddling negative regulated voltages from 5 to 24 volts.

Thermocouple: A Thermocouple is an electrical device comprising of two different conductors that forms an electrical junction at differential temperatures. It produces a temperature which is dependent on the voltage as an outcome of thermoelectric effect and this voltage is used to measure the temperatures. Fig 4. shows the basic model of the thermocouple.

Thermocouple produces signal in micro volts and measure of these requires an amplifier with low input. Thermocouple can simultaneously measure the resistance and the temperatureat the thermocouple junction which is considered as one of the most useful features of the device buzzer.

The hardware requirement used here are thermocouple, Arduino microcontroller, driver circuit and buzzer. Thermocouple is used to find the temperature of the soil. Driver circuit controls the spraying of pesticides and fertilizers. The technology used here is Embedded C. It is a high level programming language used for embedded system development.

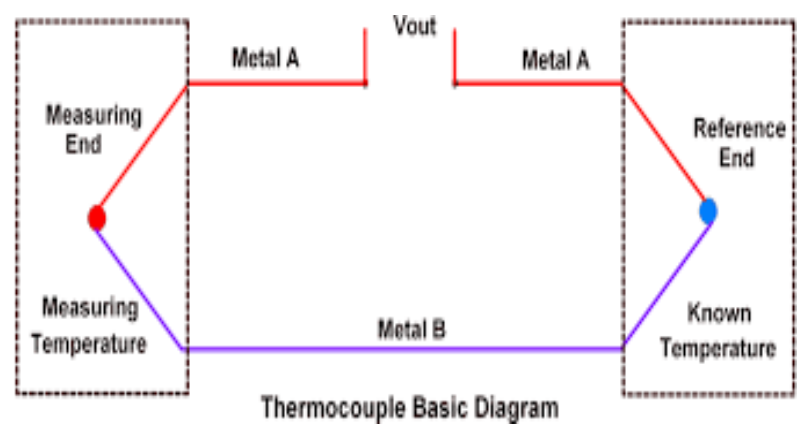

Fig.4. Basic model of thermocouple

\section{System Implementation}

This system uses embedded $\mathrm{C}$ along with Arduino software (IDE) for detecting the under soil worms. Fig 5 displays the system architecture.

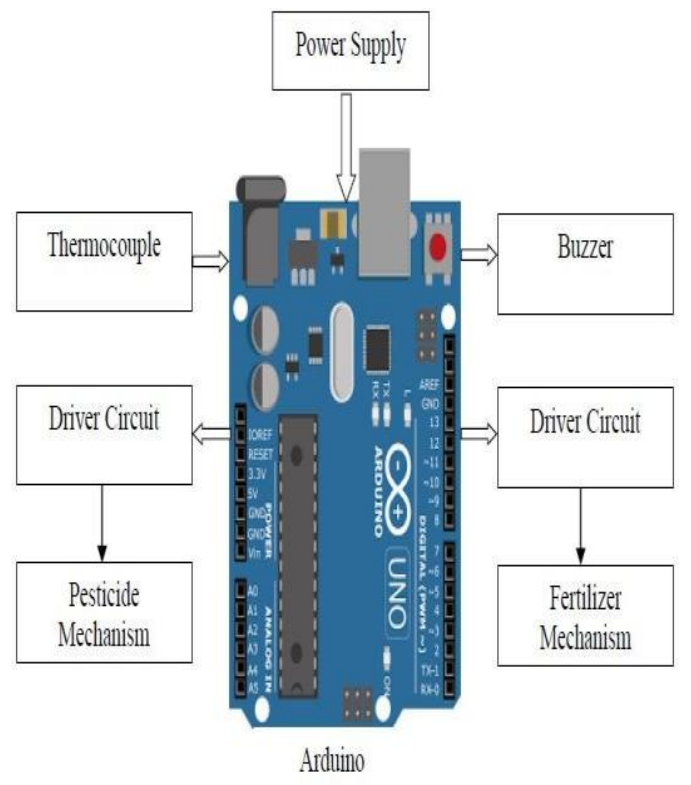

Fig.5. System Architecture

The thermocouple sensor is used to measure the temperature of the soil. When the observed temperature is normal, the driver circuit initiates the fertilizer mechanism. And the buzzer gives an alarm. The observed temperature found to be higher than that of the normal value, indicates the existence of worms under soil and the pesticide mechanism is initiated. And the buzzer gives an alert.

\section{RESULTS}

Embedded C Language is used for implementation. This language is compiled by Hi-Tech $\mathrm{C}$ compiler which uses the MPLAB IDE software. The hex code that is generated by the compiler operation is stored in the computer. This hex which is a machine level language can be assumed by the micro controller. The framework uses PICKIT2 Programmer to sear the hex code of the program into the ROM (Flash memory) of PIC16F877A. This PIC16F877A is a high performance RISC CPU machine. Fig 6 depicts the physical model of the system.

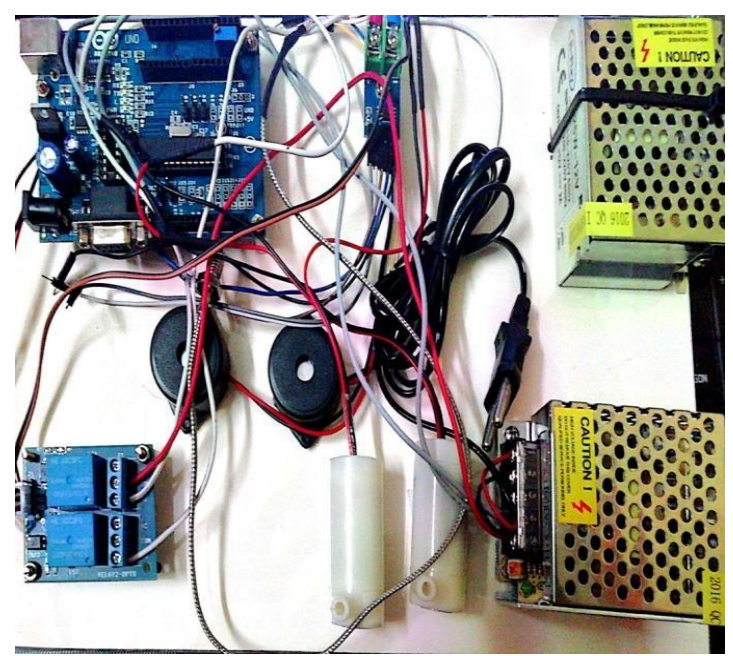

Fig.6. Physical Model of the System

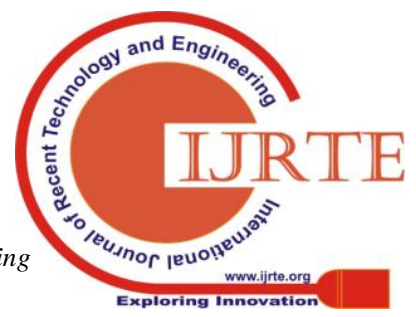




\section{CONCLUSION}

With the advancements of science and technology day to day, the proposed system has been designed to detect the crop infection by underground worm during the initial stage of the crop growth, so that the plantation crop can be prevented from getting infected in large scales. While most of the research works focuses on detecting the plant infection or disease from the symptoms that appear in the plant above the soil level, our proposed system is designed using the thermal sensor which detects the crop infection that occur below the ground level. The model that is designed is has other advantages like portability, cost effectiveness, compatibility and scalability.

\section{FUTURE WORK}

The future work would be further classify the different diseases that affect the crops for different crop species with more accuracy. The plant leaf images can be captured using drones to detect plant diseases for large farmland where manual checking is a tedious process. Drone technology will make agriculture more efficient, convenient and comfortable. It identifies various parameters such as crop stress, the healthy plants and the soil quality for the next batch of crops. Unmanned moving vehicles in agriculture are used to support to drastically escalate crop production and constantly screen the crop growth.

\section{REFERENCES}

1. Chen.Y, Zhu.H, and Ozkan.H "Development of a variableratesprayer with Laser scanning sensor to synchronize spray outputs to treestructures," Transactions of the ASABE, vol. 55, no. 3, pp. 773-781,2012.

2. Du.L, Gong.W, Shi.S, Yang.J, Sun.J, Zhu.B, and Song.S, "Estimation of rice leaf nitrogen contents based on hyperspectral LIDAR," International Journal of Applied Earth Observations and Geoinformation, vol. 44, pp. 136-143, 2016.

3. Eitel.J. U.H, Magney.T .S, Vierling.L. A, Brown.T. T, and Huggins.D. R, "LiDAR based biomass and crop nitrogen estimates for rapid, non-destructive assessment of wheat nitrogen status," Field Crops Research, vol. 159, pp. 21-32, 2014.

4. Escol`a.A, Rosell-Polo.J. R, Planas. S, Gil.E, Pomar.J, and Solanelles.F, "Variable rate sprayer. Part 1 Orchardprototype: Design, implementation and validation," Computers and Electronics in Agriculture, vol. 95, pp. 122135, 2013.

5. Foglia.M and Reina.G, "Agricultural Robot for Radicchio Harvesting," Journal of Field Robotics, vol. 23, no. 6/7, pp. 363-377, 2006

6. Gongal.A, Silwal.A, Amatya.S, Karkee.M, Zhang.Q, and Lewis.K, "Apple crop-load estimation with over-the-row machine vision system,"Computers and Electronics in Agriculture, vol. 120, pp. 26-35, 2016.

7. Ji.W, Zhao.D, Cheng.F, Xu.B, Zhang.Y, and Wang.J, "Automatic recognition vision system guided for apple harvesting robot," Computers and Electrical Engineering, vol. 38, no. 5, pp. 1186-1195, 2012.

8. Jones.H.G, Serraj.R, Loveys.B.R, Xiong.L, Wheaton.A, and Price.A. H, "Thermal infrared imaging of crop canopies for the remote diagnosis and quantication of plant responses to water stress in the field," Functional Plant Biology, vol. 36, pp. 978-989, 2009.

9. Kankare.V, Holopainen.M, Vastaranta.M, Puttonen.E, Yu.X, and Alho.P, "Individual tree biomass estimation using terrestrial laser scanning," ISPRS Journal of Photogrammetry and Remote Sensing, vol. 75, pp. 64-75, 2013.

10. Karkee.M, Adhikari.B, Amatya.S, and Zhang.Q "Identification of pruning branches in tall spindle apple trees for automated pruning,", Computers and Electronics in Agriculture, vol. 103, pp. 127-135, 2014.

11. 11. Li.W, Niu.Z, Chen.H, Li.D, Wu.M, and Zhao.W, "Remote estimation of canopy height and aboveground biomass of maize using high-resolution stereo images from a low-cost unmanned aerial vehicle system," Ecological Indicators, vol. 67, pp. 637-648, 2016.

12. 12.Mora.M, Avila.F, Carrasco-Benavides.M, Maldonado.G, Olgu'in-C'aceres.J, and Fuentes.S, “Automated computation of leaf area index from fruit trees using improved image processing algorithms applied to canopy cover digital photograpies," Computers and Electronics in Agriculture, vol. 123, pp. 195-202, 2016. 